\title{
Generation of Equally Sized Particle Plaques Using Solid-Liquid Suspensions
}

\author{
Tim Herrmann, Magnus Schröder, and Juirgen Hubbuch*
}

Institute of Biotechnology, Research Center Juelich, 52425 Juelich, Germany

\begin{abstract}
A device is presented for the generation of equally sized plaques of sensitive particles in a 96-well format. The resulting particle plaques can be used for the measurement of adsorption isotherms and uptake kinetics in protein chromatography or for immobilization reactions. The particle plaques are formed from suspensions with a vacuum device that is designed as a reusable sandwich module. The particles are retained by a mesh while the solvent is removed by the vacuum. As most particles used for protein chromatography are sensitive to mechanical stress and dehydration, the vacuum device is gentle enough to allow the use of these particles, thus eliminating the uncertainty of slurry preparation and pipetting. Apparatus characteristics and preparation procedures are described precisely. The physical intactness of the particles after the preparation procedure is proved by microscopic analysis. Data on the uniformity of the obtained resin plaques with respect to the reproducibility of their adsorption performance is given. Finally, adsorption isothermal and kinetic data of BSA on an ordinary HIC system obtained by highthroughput measurements are shown as an application example.
\end{abstract}

\section{Introduction}

Chromatography is a column-based separation method where particles are most frequently used as stationary phases. Column performance is influenced by thermodynamic and kinetic parameters. Measurement of adsorption isotherms and kinetics using finite bath experiments build the basis for a rational process design that facilitates the setup of purification schemes. Both systems have in common that mass balances have to be set up in order to obtain the respective parameters.

This said, many particles used for protein chromatography, especially those based on natural polymers, are sensitive to mechanical stress and dehydration. Other systems that share theses properties are, for example, particles used for enzyme immobilization. The high amount of variable parameters in chromatographic process characterization or enzymatic conversion studies and mostly restricted feedstock availability calls for an intensive use of finite bath studies substituting time and feedstock consuming column optimization procedures.

This approach is suitable for sample volume reduction and process automation to reduce feedstock consumption and to increase the data throughput, a general trend usually referred to as high-throughput screening (HTS). HTS assays for bioprocess optimization experience a growing acceptance $(1,2)$ and are commonly performed in homogeneous suspension in microtiter plates to analyze solute concentration or solution parameters $(3-5)$. Robotic systems have been constructed to automatically process microtiter plates in conjunction with unit operations such as mixing or filtration and suitable detection systems. Determination of mass balances, however, requires the precise knowledge of the amount of stationary phase, the reaction volume, and solute concentrations.

Current technology is capable of precise fluid transfer in the microliter regime, especially in robotic systems, but there is still a challenge to control the volume of particles in suspension-

* To whom correspondence should be addressed. Ph: +49-2461-616044. Fax: +49-2461-61-3870. Email: j.hubbuch@fz-juelich.de. based systems. While there are existing technologies for dry particles that can be pressed to pellets or weighted, particles used for protein purification or for immobilization reactions often cannot be dispensed in this way. Some authors use pipetting to dispense their particle suspension (6), but this approach is hampered by the fact that the mixing of the particle slurry is not ensured. This leads to a nonconstant particle concentration and size distribution in each sample that is being pipetted.

The presented vacuum manifold device (VMD) circumvents the problems that arise with pipetting or drying by simply filling up a preformed gap in a grid plate with particles under vacuum. The particles are filled into the device in a suspension and are in a moist state when the particle plaques are formed so that their full structural integrity is ensured. The device is suited for 96-well plate applications by virtue of its design. Alternatively to pneumatic forces, centrifugation can be used (7) for slurry conveyance. With the vacuum manifold device highthroughput screening in protein chromatography or in immobilization reactions becomes easier and more precise.

\section{Materials and Methods}

Vacuum Manifold Device. There are two model versions, prototype and serial model, of the VMD presented in this paper. The prototype version, which was developed at our Institute, was licensed by Atoll (8) and developed into a serial model (MediaScout Resi Quot (8)). Both model versions have a similar setup consisting of four main parts (see Figure 1, parts 1, 3, $5 / 6 / 10,11$ ) that can be screwed together but vary in the materials used for the construction of some parts.

The top part (Figure 1, part 1) functions as a slurry reservoir and has 96 openings. It is made of plexiglass in the prototype version and of anodically oxidized Duralumin in the serial version. The spacing between the 96 openings fit to a standard 96-well plate. The bottom of the reservoir is surrounded by an O-ring (Figure 1, part 9). The second part (Figure 1, parts 5/6/ 10) forms the grid, where the adsorber plaques are generated. 

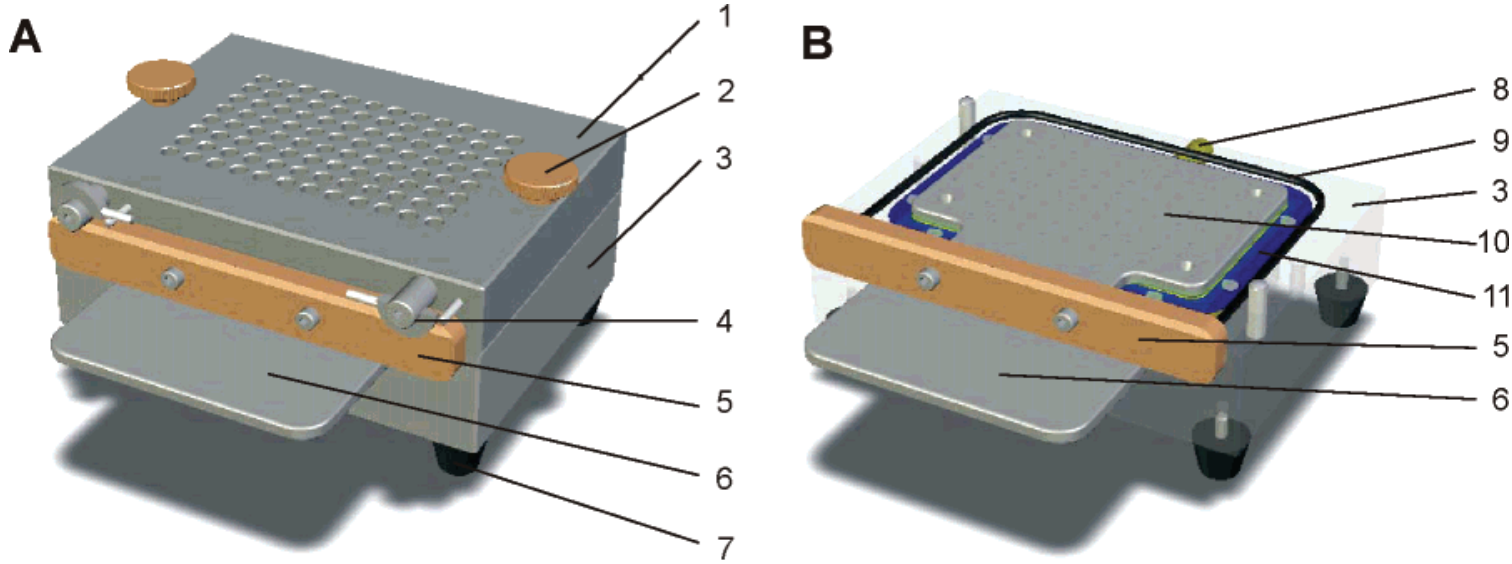

Figure 1. Diagram of fully assembled MediaScout ResiQuot unit (A) and expanded diagram of the unit with the upper manifold removed (B). Part description: (1) upper manifold device, (2) knurled assembly screws, (3) lower manifold plate, (4) insertion clamps, (5) stopper plate, (6) slider handle, (7) feet, (8) vacuum connection, (9) gasket seal, (10) perforated plate, (11) frit holder with stainless steel frit. Published with permission of Atoll (8).

In the prototype version the grid plate is a flat piece of Plexiglas with 96 holes drilled with a diameter of 1.5 or $2 \mathrm{~mm}$ each, whereas it is made of stainless steel with hole diameters of 1.4 $\mathrm{mm}$ in the serial model. The 96 holes are aligned with the 96 openings of the slurry reservoir. At one end of the grid plate a handle is located with its inside being surrounded by an O-ring that ensures a tight fit between the top and bottom piece of the VMD. The frit holder (Figure 1, part 11) is positioned under the grid plate and fits into indentations of the bottom piece (Figure 1, part 3). The frit consists of stainless steel with a thickness of $0.07 \mathrm{~mm}$ and a pore size of $10 \mu \mathrm{m}$. Finally the bottom piece (Figure 1, part 3) is a rectangular block that holds the withdrawn liquids and has an adaptor (Figure 1, part 8) to which a vacuum pump is connected. Materials used for the bottom piece are analogue to those used for the top part.

Generation of Plaques. To generate 96 equally sized plaques, a slurry of particles is filled in all openings of the reservoir of the VMD. After that a vacuum pump is connected to the device and is activated for $15 \mathrm{~min}$. The slurry is sucked into the grid plate, and the particles are retained by the filter mesh. A 15 min vacuum ensures a consistent packing density and humidity of the plaques which are then ready for immediate use. To obtain them the screws of the device have to be removed and the grid plate has to be pulled out of the device with the attached handle. A clean grid plate with its 96 holes filled with particles is obtained by integrated wipers in the serial version and by wiping it off manually when working with the prototype. The particles can then be pushed out of the holes quantitatively with a complementary pin or with a 96-well format removal tool aligned to the 96 holes. The fit tolerance between the pins and the holes is 0.857 to 1 in case of the stainless steel grid of the serial model and 1 to 1 for the plexiglass grid of the prototype model.

Characterization of Plaques. The uniformity of particle plaques was characterized indirectly by measuring the reproducibility of the plaques adsorption capacities due to a constant adsorber amount per plaque. A cation exchange mechanism with sulfopropyl ligands as functional groups and lysozyme (Sigma, Taufkirchen, Germany) as adsorptive in a sodium acetate buffer at pH 5.0 was selected as working platform. Experiments were conducted in batch mode in 96 deep well plates. Different kinds of adsorber backbones were used (see Table 1) to determine possible dependencies of the plaque forming performance on the material used.
Table 1. Adsorber Materials Used for Plaque Preparation

\begin{tabular}{llcl}
\hline \multicolumn{1}{c}{ product } & \multicolumn{1}{c}{$\begin{array}{c}\text { adsorber } \\
\text { backbone }\end{array}$} & $\begin{array}{c}\text { bead diam }^{a} \\
{[\mu \mathrm{m}]}\end{array}$ & \multicolumn{1}{c}{ manufacturer } \\
\hline S Ceramic Hyper D & ceramic & 50 & Pall $^{b}$ \\
SP Sepharose FF & Sepharose & $90^{e}$ & GE Healthcare $^{c}$ \\
Streamline SP & quartz-Sepharose & $200^{e}$ & GE Healthcare $^{c}$ \\
Toyopearl 650 M SP & methacrylate & 65 & Tosoh $^{d}$
\end{tabular}

${ }^{a}$ According to manufacturer. ${ }^{b}$ Pall GmbH, Dreieich, Germany. ${ }^{c} \mathrm{GE}$ Healthcare Europe GmbH, Munich, Germany. ${ }^{d}$ Tosoh Biosciences, Stuttgart, Germany. ${ }^{e}$ Mean bead diameter.

Stock solutions with different concentrations of lysozyme dissolved in $20 \mathrm{mM}$ sodium acetate buffer at $\mathrm{pH} 5.0$ at room temperature were prepared. For each adsorber type $2 \times 96$ plaques with a cylinder volume of $7.7 \mu \mathrm{L}$ were produced using the serial model. In addition, $2 \times 96$ plaques with a cylinder volume of $8.0 \mu \mathrm{L}$ and $2 \times 96$ plaques with a cylinder volume of $15.1 \mu \mathrm{L}$ were produced with SP Sepharose FF using the prototype model with two respective grids. Finally, a 1:4 slurry of SP Sepharose FF was prepared in a cylinder resulting in a desired cylinder volume of $15 \mu \mathrm{L}$ of adsorber material per pipetting step when $60 \mu \mathrm{L}$ was pipetted manually.

The preparation procedure described above resulted in a double series of seven different 96er sets of adsorber aliquots. They were dispensed in deep well plates, where one pair of each series was incubated with two different protein concentrations. On a robotic workstation (Tecan Deutschland $\mathrm{GmbH}$, Crailsheim, Germany) $500 \mu \mathrm{L}$ of protein solution was transferred into each well, and the plate was tightly capped and incubated overnight on a horizontal shaker. The mixing behavior in these microliter scale vessels had been investigated (10), and thus sufficient adjustments were made to provide complete mixing. Following incubation the plate was centrifuged for $2 \mathrm{~min}$ at $50 \mathrm{~g}$ and the supernatant subsequently analyzed. Liquid transfer and UV measurements of the supernatants were conducted via an automated script on the robotic workstation employing 96well UV plates (Greiner UV star, Greiner Bio-One $\mathrm{GmbH}$, Solingen, Germany) and a photometer (Genios Pro, Tecan Deutschland $\mathrm{GmbH}$, Crailsheim, Germany). This procedure was applied identically to the two series of protein adsorber systems as well as to the different reference protein solutions.

Microscopic Analysis of Single Particles after Plaque Production. After the plaques were analyzed as described above 16 wells of each 96 deep well plate were chosen arbitrarily. The particles were manually mixed with the remaining liquid, 
Table 2. Summary of Adsorber Aliquots Generated; for Each Line $2 \times 96$ Aliquots Were Produced

\begin{tabular}{llrc}
\hline \multicolumn{1}{c}{ apparatus $^{a}$} & \multicolumn{1}{c}{$\begin{array}{c}\text { grid } \\
\text { vol }^{a}[\mu \mathrm{L}]\end{array}$} & $\begin{array}{c}\text { adsorber } \\
\text { vol }^{c}[\mu \mathrm{L}]\end{array}$ \\
\hline 8-channel pipet & SP Sepharose FF & 15.0 & 9.5 \\
prototype model, Grid 1 & SP Sepharose FF & 8.0 & 5.0 \\
prototype model, Grid 2 & SP Sepharose FF & 15.1 & 9.5 \\
serial model, Grid 1 & SP Sepharose FF & 7.7 & 4.8 \\
serial model, Grid 1 & S Ceramic Hyper D & 7.7 & 4.8 \\
serial model, Grid 1 & Streamline SP & 7.7 & 4.8 \\
serial model, Grid 1 & Toyopearl 650 M SP & 7.7 & 4.8
\end{tabular}

${ }^{a}$ See Materials and Methods, Vacuum Manifold Device. ${ }^{b}$ See Table 1 for further reference. ${ }^{c}$ Calculated from the grid volume with a packed bed porosity factor of 0.37 proposed by Brauer (9).

and $50 \mu \mathrm{L}$ of the slurry was pipetted on a microscope slide. The particles were analyzed under a light microscope with a water immersion objective lens (63-fold magnification, NA: 1.2, Carl Zeiss AG, Jena, Germany).

Application Examples. Adsorption Isotherms. adsorption isotherms were measured in batch experiments with varying starting protein concentrations in 96 deep well plates. After the particle plaques were dispensed into the deep well plate, 800 $\mu \mathrm{L}$ of protein solution was transferred into the wells and the plate was tightly capped. The deep well plate was incubated for $2 \mathrm{~h}$ on a horizontal shaker after which the plate was centrifuged for $2 \mathrm{~min}$ at $50 \mathrm{~g}$. Together with the analysis of the supernatant before and after incubation the adsorption isotherm was constructed from a mass balance (eq 1)

$$
q_{\text {eq }}=\frac{V_{\text {fluid }} \cdot\left(c_{\text {init }}-c_{\text {eq }}\right)}{V_{\text {adsorber }}}
$$

with $q_{\text {eq }}$ as adsorbed protein concentration, $V_{\text {system }}$ as total reaction volume, $V_{\text {adsorber }}$ as stationary phase volume, and $c_{\text {init }}$ and $c_{\mathrm{eq}}$ as starting and equilibrium protein concentration, respectively.

Adsorption Kinetics. Kinetic uptake experiments were conducted employing one adsorber plaque and $800 \mu \mathrm{L}$ of protein solution of fixed initial concentrations. After all reaction tubes were filled with one plaque the respective protein solutions were added at different time points and afterward incubated on a horizontal shaker. All incubation reactions were stopped simultaneously by a $30 \mathrm{~s}$ centrifugation and subsequent transfer of the supernatant to a new reaction tube. The whole procedure took less than $2 \mathrm{~min}$. Protein concentration in the supernatant before and after the addition of the adsorber plaque was determined and normalized (eq 2)

$$
c_{\text {norm }}=\frac{c_{\text {init }}-c_{\text {final }}}{c_{\text {init }}}
$$

with $c_{\text {norm }}, c_{\text {init }}$, and $c_{\text {final }}$ being the normalized, initial and final protein concentration in the supernatant.

\section{Results and Discussion}

Characterization and Scalability of Particle Plaques. Plaque Production. Two series of $7 \times 96$ adsorber aliquots were prepared by means of different materials (see Table 1) and different devices. A summary is shown in Table 2. The volume of the adsorber beads being packed in the grid cylinders can be approximated using a packed bed porosity factor of 0.37 proposed by Brauer (9).

Depending on the suitable amount of resins in an experimental study the amount of adsorber material obtained can be varied either by using multiple resins of a distinct size per well or by

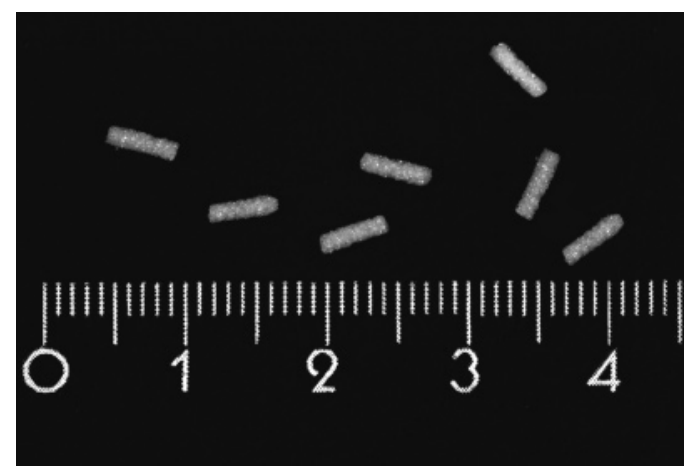

Figure 2. Adsorber plaques witch a cylinder volume of $7.7 \mu \mathrm{L}$ made of SP Sepharose FF are shown together with a scalebar (major increments in $\mathrm{cm}$ ).

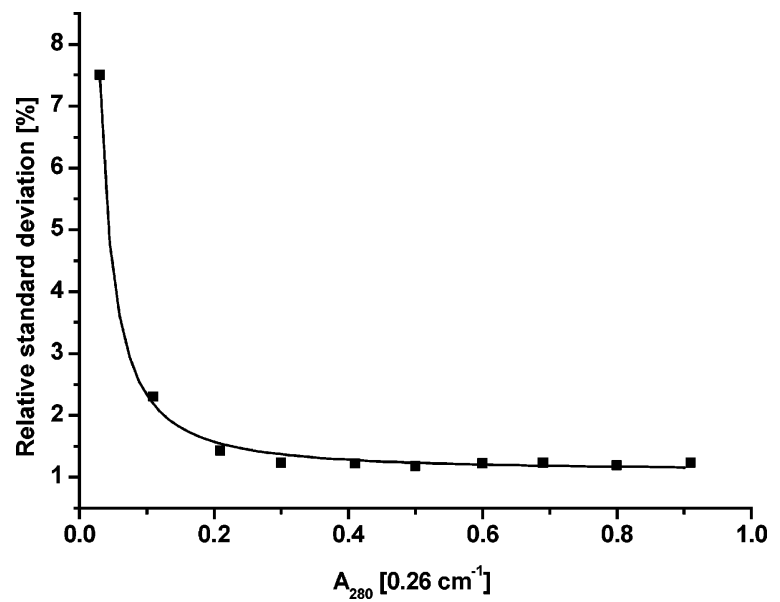

Figure 3. Relative standard deviations of nine lysozyme reference solutions and the buffer used are plotted against their mean absorption value (abscissa). Lysozyme is dissolved in different concentrations in $20 \mathrm{mM}$ sodium acetate buffer, $\mathrm{pH} 5$ at room temperature. Each solution was measured 96 times on a UV plate employing an automated script on a robotic workstation. The data set can be described through an extended Freundlich model $y=a+b \times x^{c}$ with a stability index of 0.9983 .

simply using a grid plate with larger/smaller openings as shown above. Figure 2 shows particle plaques with a cylinder volume of $7.7 \mu \mathrm{L}$ made of SP Sepharose FF with the serial model. The dimensions of the openings, however, have to be within the mechanically feasible range of plaque preparation and handling, which corresponds to a plaque volume of $5-50 \mu \mathrm{L}$ with the apparatus currently used. Additionally, the multiwell format can also be adapted to other well numbers such as the commercially available 384 well format.

$\boldsymbol{U} \boldsymbol{V}$ Measurements. The adsorption capacities of the 14 different sets of adsorber aliquots were determined as described in Materials and Methods. To make the supernatant UV absorption signals as precisely ascribable to the plaque amount deviation as possible, equilibrium adsorption capacities were determined. They are preferable in comparison to dynamic adsorption measurements, which have a potential higher systematic error due to the time dependency of the measurements. Experimental conditions were deliberately set to provide complete saturation of the adsorber particles. For each set a mean extinction value and its standard deviation were calculated from the 96 single measurements. The signals obtained at $280 \mathrm{~nm}$ lie in a range of $0.10 \times\left(0.26 \mathrm{~cm}^{-1}\right)$ to $1.05 \times\left(0.26 \mathrm{~cm}^{-1}\right)$ and show a direct proportionality to the respective protein concentration resulting in a calibration curve with a stability index of 0.9994 (data not presented). UV measurement conditions are identical for supernatant as well as for reference protein solutions 
Table 3. UV Measurements of 14 Sets of Adsorber Aliquots Incubated with Different Stock Solutions ${ }^{i}$

\begin{tabular}{|c|c|c|c|c|c|c|c|}
\hline apparatus $^{a}$ & adsorber material $^{b}$ & $\begin{array}{c}V_{\text {adsorber }^{c}} \\
{[\mu \mathrm{L}]}\end{array}$ & $\begin{array}{l}c_{\text {protein.stock }}{ }^{d} \\
{\left[\mathrm{mg} \mathrm{mL}^{-1}\right]}\end{array}$ & $\begin{array}{c}A_{280, \text { mean }^{e}} \\
{\left[0.26 \mathrm{~cm}^{-1}\right]}\end{array}$ & $\begin{array}{c}\mathrm{RSD}_{\mathrm{UV}, \text { super }^{f}} \\
{[\%]}\end{array}$ & $\begin{array}{c}\mathrm{RSD}_{\mathrm{UV}}, \mathrm{ref}^{\mathrm{g}} \\
{[\%]}\end{array}$ & $\begin{array}{c}\mathrm{RSD}_{\text {plaques }}{ }^{h} \\
{[\%]}\end{array}$ \\
\hline PT & Seph FF & 9.5 & 2.5 & 0.42 & 42.42 & 1.26 & 6.08 \\
\hline PT & Seph FF & 9.5 & 3.0 & 0.75 & 24.02 & 1.16 & 6.10 \\
\hline PM & Seph FF & 5.0 & 2.0 & 0.68 & 4.69 & 1.17 & 1.97 \\
\hline PM & Seph FF & 5.0 & 2.5 & 1.01 & 3.26 & 1.13 & 1.96 \\
\hline PM & Seph FF & 9.5 & 2.5 & 0.42 & 8.36 & 1.26 & 1.12 \\
\hline PM & Seph FF & 9.5 & 3.0 & 0.75 & 4.65 & 1.16 & 1.08 \\
\hline SM & Seph FF & 4.8 & 1.5 & 0.38 & 6.39 & 1.28 & 1.56 \\
\hline SM & Seph FF & 4.8 & 2.0 & 0.71 & 3.53 & 1.17 & 1.54 \\
\hline SM & Cer HD & 4.8 & 1.0 & 0.21 & 9.77 & 1.53 & 1.80 \\
\hline SM & Cer HD & 4.8 & 2.0 & 0.86 & 2.62 & 1.15 & 1.79 \\
\hline SM & Streaml & 4.8 & 1.0 & 0.31 & 5.96 & 1.35 & 1.96 \\
\hline SM & Streaml & 4.8 & 2.0 & 0.96 & 2.19 & 1.14 & 1.98 \\
\hline SM & Toyop & 4.8 & 1.0 & 0.37 & 3.78 & 1.29 & 1.72 \\
\hline SM & Toyop & 4.8 & 2.0 & 1.02 & 1.70 & 1.13 & 1.71 \\
\hline
\end{tabular}

${ }^{a}$ Apparatuses used: $\mathrm{PT}=8$-channel pipet, $\mathrm{PM}=$ prototype model, $\mathrm{SM}=$ serial model. ${ }^{b}$ Adsorber material used: $\mathrm{Seph} \mathrm{FF}=\mathrm{SP}$ Sepharose $\mathrm{FF}, \mathrm{Cer} \mathrm{HD}$ $=\mathrm{S}$ Ceramic Hyper D, Streaml $=$ Streamline SP, Toyop $=$ Toyopearl $650 \mathrm{M} \mathrm{SP} .{ }^{c}$ Adsorber volume calculated from cylinder volume of the grid holes times the packed bed porosity factor (0.37) proposed by Brauer (9). ${ }^{d}$ Concentration of the stock solutions: $x$ mg lysoyme in $20 \mathrm{mM}$ sodium acetate buffer, $\mathrm{pH}$ 5 at room temperature. ${ }^{e}$ Mean absorption values at $280 \mathrm{~nm}$ calculated from 96 measurements. ${ }^{f}$ Relative standard deviations of absorption values at $280 \mathrm{~nm}$ calculated from 96 measurements. ${ }^{g}$ Relative standard deviations caused by error sources (i)-(iii), derived from Figure $3 .{ }^{h}$ Relative standard deviations calculated applying eq 5. ${ }^{i}$ Lysozyme was dissolved in different concentrations ( $\left.c_{\text {protein.stock }}\right)$ in $20 \mathrm{mM}$ sodium acetate buffer, $\mathrm{pH} 5$ at room temperature. Adsorber aliquots were generated using different apparatuses (apparatus $/ V_{\text {adsorber }}$ ) and materials (adsorber material). The distribution of the 96 absorption values is characterized by a mean value $\left(A_{280, \text { mean }}\right)$ and a relative standard deviation (RSDUV,super). RSDs of the adsorber amount per plaque can calculated from eq 5 .
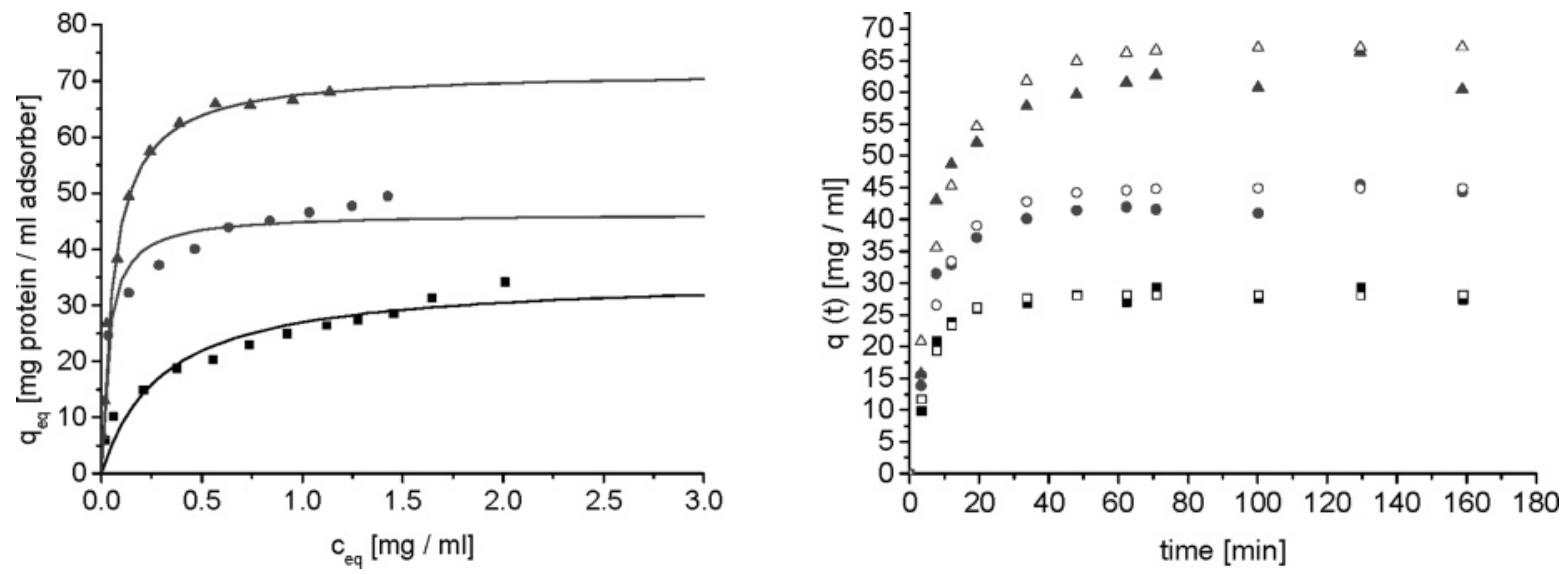

Figure 4. Adsorption isotherms (left) and kinetics (right) of bovine serum albumin (BSA) on butyl-Sepharose FF in $0.02 \mathrm{M}$ potassium phosphate buffer, pH 7 with additional $0.5 \mathrm{M}(\boldsymbol{\square}), 1.0 \mathrm{M}(\bullet)$, or $1.5 \mathrm{M}(\boldsymbol{\Delta})\left(\mathrm{NH}_{4}\right)_{2} \mathrm{SO}_{4}$. Left: The protein concentration on the stationary phase $\left(q_{\mathrm{eq}}\right)$ is plotted against the corresponding fluid phase concentration $\left(c_{\mathrm{eq}}\right)$. Model parameters $K_{\mathrm{a}}$ and $q_{\mathrm{m}}$ are fitted with the Langmuir model (straight lines). Right: The time-dependent adsorber capacity is plotted against the time $(t)$. Model parameter $k_{\mathrm{a}}$ (hollow symbols) is fitted with the Langmuir model.

and are prone to three sources of measurement errors: (i) pipetting error when transferring the $100 \mu \mathrm{L}$ of protein solution from the 96 deep well plate to the $96 \mathrm{UV}$ plate, resulting in different heights of the fluid meniscus (no path length correction was applied); (ii) material inconsistencies of the UV transparent bottom of the UV plate; and (iii) inherent errors of the photometer due to deviations from the optimal beam path. The sum of errors (i)-(iii), further referred to as $\epsilon_{\mathrm{UV} \text {,reference, }}$ can be quantified by calculating the standard deviations from 96 single measurements of each reference protein solution.

The different reference protein solutions were measured as described in Materials and Methods. Mean value, standard deviation, and relative standard deviation (RSD) as quotient of both were calculated (see Figure 3). The resulting data set can be described through an extended Freundlich model (eq 3):

$$
y=a+b \times x^{c}
$$

The stability index of the fit is 0.9983 , the values of the parameters are $a=1.07722, b=0.05576$, and $c=-1.35394$. The RSD is highest $(7.5 \%)$ for the pure buffer solution with an absorption coefficient of $0.03 \times\left(0.26 \mathrm{~cm}^{-1}\right)$. It declines exponentially toward a constant value of about $1.2 \%$ within the region of interest. Thus, systematic measurement errors mainly caused by material inconsistencies of the UV plate prevail in low absorbingregions, which are overshadowed by very similar random errors in the higher absorbing regions. Therefore, the lysozyme concentrations of the stock solutions used for the plaque measurements were chosen so that the absorption signals of the supernatants after adsorption lay in the range between $0.2 \times\left(0.26 \mathrm{~cm}^{-1}\right)$ and $1.0 \times\left(0.26 \mathrm{~cm}^{-1}\right)$

When analyzing the supernatant protein solutions of the adsorption experiments, however, two additional error sources have to be considered: (iv) pipetting error, $\epsilon_{\text {fluid, }}$, when dispensing the $500 \mu \mathrm{L}$ of protein solution into the deep well plate and (v) a varying adsorber amount per plaque, $\epsilon_{\text {adsorber }}$. The sum of errors (i) $-(\mathrm{v})$, further referred to as $\epsilon_{\mathrm{UV} \text {,supernatant }}$ can be quantified by calculating the standard deviations from 96 single measurements of each supernatant of the adsorption experiments. Thus, construction of the mass balances (eq 1) leads to (eq 4):

$$
\begin{gathered}
c_{\text {eq }}=c_{\text {init }}-q_{\text {eq }} \frac{V_{\text {adsorber }}+\epsilon_{\text {adsorber }}}{V_{\text {fluid }}+\epsilon_{\text {fluid }}}+\epsilon_{\mathrm{UV}, \text { supernatant }}= \\
c_{\text {reference }}+\epsilon_{\mathrm{UV}, \text { reference }}
\end{gathered}
$$

with $c_{\text {init }}, c_{\mathrm{eq}}, c_{\text {reference }}$ as initial, equilibrium, and reference protein 
Table 4. Coefficients of Determination for Langmuir Parameter Fits of Data Presented in Figure $4^{a}$

\begin{tabular}{ccc}
\hline & \multicolumn{2}{c}{ coefficient of determination } \\
\cline { 2 - 3 }$c_{\left(\mathrm{NH}_{4}\right)_{2} \mathrm{SO}_{4}}[\mathrm{M}]$ & isotherms [1] & kinetics [1] \\
\hline 0.5 & 0.9098 & 0.9648 \\
1.0 & 0.9050 & 0.9358 \\
1.5 & 0.9936 & 0.9189
\end{tabular}

${ }^{a}$ Langmuir parameters $K_{\mathrm{a}}$ and $q_{\mathrm{m}}$ were derived from isothermal data, parameter $k_{\mathrm{a}}$ from kinetic data.

fluid phase concentrations, $q_{\mathrm{eq}}$ as equilibrium protein concentration on the adsorber material, $V_{\text {adsorber }}, V_{\text {fluid }}$ as adsorber and fluid phase volume, and $\epsilon_{\text {analytics }}, \epsilon_{\text {fluid }}, \epsilon_{\text {adsorber }}, \epsilon_{\mathrm{UV} \text {,supernatant, }}$, and $\epsilon_{\mathrm{UV}, \text { reference }}$ as respective errors (absolute values). Assuming homogeneous binding conditions for each adsorber type and applying the Gaussian error propagation law, $\epsilon_{\text {adsorber }}$ can be calculated for similar mean adsorption values, i.e., $c_{\text {reference }}=$ $c_{\text {eq }}($ eq 5):

$$
\begin{aligned}
& \epsilon_{\text {adsorber }}= \\
& \sqrt{\left(\epsilon_{\mathrm{UV}, \text { supernatant }}^{2}-\epsilon_{\mathrm{UV}, \text { reference }}^{2}\right) \frac{V_{\text {fluid }}^{2}}{q_{\mathrm{eq}}^{2}}-\frac{V_{\text {adsorber }}^{2}}{V_{\text {fluid }}^{2}} \epsilon_{\text {fluid }}^{2}}
\end{aligned}
$$

The pipetting error $\epsilon_{\text {fluid }}$ of the 8-channel pipet was found to be $0.4 \%$ (data not presented). The results obtained for the different protein adsorber systems are summarized in Table 3.

Within the absorption range mentioned above the RSD of the adsorber volume can be quantified. Pipetting the adsorber slurry manually leads to almost $4 \%$ greater variations. All four adsorber backbones are suitable for plaque preparation, with Sepharose being the most and streamline the least handsome. Increasing the plaque volume leads to a decrease in adsorber volume variation. By using the VMD the accuracy of plaque production is $\geq 98 \%$.

Microscopic Analysis of Single Particles after Plaque Production. Single particles were analyzed as described in Materials and Methods. No friction could be detected (data not shown).

Application Examples: Adsorption Isotherms/Kinetics. By using the VMD generated adsorber plaques, consistent adsorption isotherms and kinetics can be measured with good accuracy. Figure 4 shows a HIC adsorber-buffer system commonly applied in chromatographic processes with BSA as model protein. The data sets are described with the time (in-)dependent version of the Langmuir model (eq 6):

$$
\frac{\mathrm{d} q}{\mathrm{~d} t}=k_{\mathrm{a}} c\left(q_{\mathrm{m}}-q\right)-k_{\mathrm{d}} q ; \quad q_{\mathrm{eq}}=\frac{q_{\mathrm{m}} K_{\mathrm{a}} c_{\mathrm{eq}}}{1+K_{\mathrm{a}} c_{\mathrm{eq}}} ; \quad K_{\mathrm{a}}=\frac{k_{\mathrm{a}}}{k_{\mathrm{d}}}
$$

with $q, q_{\mathrm{m}}$, and $q_{\mathrm{eq}}$ as time dependent, maximum, and equilibrium capacity, $c$ and $c_{\text {eq }}$ as time dependent and equilibrium fluid phase concentration, $t$ as time, $K_{\mathrm{a}}$ as adsorption constant, $k_{\mathrm{a}}$ as volume specific rate constant of adsorption, and $k_{\mathrm{d}}$ as rate constant of desorption.

The isotherms presented resulted from singular measurements and show a good correlation. Isothermal data was used to fit the Langmuir parameters $K_{\mathrm{a}}$ and $q_{\mathrm{m}}$, and kinetic data was used to fit the parameter $k_{\mathrm{a}}$. The coefficients of determination are shown in Table 4.

Typical maximum adsorber capacities lie in a range of $0-120$ mg protein per milliliter of adsorber, which corresponds to a capacity of $0-0.94 \mathrm{mg}$ protein per adsorber plaque. Thus, at most $1 \mathrm{mg}$ of protein has to be consumed per well or data point, respectively. For the isotherms shown in Figure 3, $40 \mathrm{mg}$ of protein was consumed. If 12 data points are desired to characterize one isotherm, $8 / 4$ isotherms can be obtained in singular/duplicate measurements within $4 \mathrm{~h}$ total work time including all preparative work. For the generation of kinetic data material and time expenses are comparable to the isotherm measurements.

\section{Conclusion}

The Vacuum Manifold Device is a useful tool to produce equally sized amounts of particles that are sensitive to mechanical stress and dehydration by forming them into plaques. For most commonly applied protein chromatography resins the variation of the adsorber amount per plaque is less than $2 \%$ while their structural and functional integrity is ensured.

\section{Acknowledgment}

This work was financed by the German Research Community (DFG). Special thanks go to Dr. Eric von Lieres for his support on the subject of error propagation. Thanks to Thomas Rosenbaum for taking the photograph of the adsorber plaques (Figure 2). Finally, Arthur Susanto is greatly acknowledged for his support in data processing.

\section{References and Notes}

(1) Lye, G. J.; Ayazi-Shamlou, P.; Baganz, F.; Dalby, P. A.; Woodley, J. M. Accelerated design of bioconversion processes using automated microscale processing techniques. Trends Biotechnol. 2003, 21, 2937.

(2) Welch, C. J.; Shaimi, M.; Biba, M.; Chilenski, J. R.; Szumigala, R. H.; Dolling, U., Jr.; Mathre, D. J.; Reider, P. J. Microplate evaluation of process adsorbents. J. Sep. Sci. 2002, 25, 847-850.

(3) Burbaum, J.; Sigalt, N. H. New technologies for high-throughput screening. Curr. Opin. Chem. Biol. 1997, 1, 72-78.

(4) Sundberg, S. A. High-throughput and ultra-high-throughput screening: solution- and cell-based approaches. Curr. Opin. Biotechnol. 2000, 11, 47-53.

(5) Hertzberg, R. P.; Pope, A. J. High-throughput screening: new technology for the 21st century. Curr. Opin. Chem. Biol. 2000, 4, $445-451$.

(6) Rege, K.; Ladiwala, A.; Tugcu, N.; Breneman, C. M.; Cramer, S. M. Parallel screening of selective and high-affinity displacers for proteins in ion-exchange systems. J. Chromatogr. A 2004, 1033, 9-28.

(7) Linden T. Untersuchungen zum Inneren Transport bei der Proteinadsorption an poröse Medien mittels konfokaler Laser Raster Mikroskopie. Ph.D. Thesis, Heinrich Heine University Duesseldorf, Germany, 2001.

(8) Atoll GmbH, Ettishofer Str. 10, 88250 Weingarten, Germany. Further information: http://www.atoll-ger.de

(9) Brauer, H. Grundlagen der Einphasen- und Mehrphasenströmungen. Verlag Sauerländer: Aarau, Frankfurt am Main, Germany, 1971.

(10) Bensch, M.; Schulze-Wierling, P.; von Lieres, E.; Hubbuch, J. High throughput screening of chromatographic phases for rapid process development. Chem. Eng. Technol. 2005, 28 (11), 12741284.

Accepted for publication March 23, 2006.

BP050296I 